4. C.P. Reghunadhan Nair and G. Clouet, IMS Rev. Macromol. Chem. Phys. C31 (1991) p. 311. 5. P. Marie and Y. Rouault, "Interfacial Tension between Immiscible Molten Polymers. Time, Temperature and Molecular Weight Dependence," Macromolecules, in press.

6. S.R. Jin, J.M. Widmaier, and G.C. Meyer, Polym. 29 (1988) p. 346.

7. G. Weill, JMS Rev. Macromol. Chem. Phys. A 27 (1990) p. 1769.

8. D. Graebling, D. Froelich, and R. Muller, J. Rheol. 33 (1989) p. 1283
9. J.F. Palierne, Rheologica Acta 29 (1990) p. 204 10. M. Lambla and M. Seadan, Polym. Eng. Sci. 32 (22) (1992) p. 1687.

11. J. Perez, Physique et Mécanique des Polyméres Amorphes, (Lavoisier, Paris, 1992).

12. O. Mauzac and R. Schirrer, J. Mater. Sci. 25 (1990) p. 5125.

13. J.C. Wittmann and B. Lotz, Prog. Polym. Sci. 15 (1990) p. 909.

14. A. Thierry, B. Fillon, C. Strauṕ, B. Lotz, and J.C. Wittmann, Prog. Colloid Polym. Sci. 87 (1992) p. 28
Gilbert Weill, professor of physics at University Louis Pasteur, Strasbourg, France, is head of the Institute C. Sadron. His main interests are in chain conformations, interactions, and dynamics with special emphasis on polyelectrolytes and solid-state NMR.

Institute C. Sadron (CRM-EAHP)

CNRS E Université Louis Pasteur

6 , rue Boussingault 67083

Strasbourg Cédex France

\title{
UPCOMING CONFERENCES
}

\section{APS Materials Physics Division Plans Focused Sessions for March Meeting}

The American Physical Society's Division of Materials Physics has planned a series of 19 focused sessions for the APS Meeting in Pittsburgh, Pennsylvania, March 21-25, 1994. In the focused sessions, contributed papers are carefully organized around particular topics to make them more functional. A focused session typically begins with an invited paper chosen to set the stage for the contributed papers and discussions.

Focused sessions are planned on the following topics:

- Theory of Materials - advances in theoretical modeling and computational studies of materials; calculations for technologically important compounds; diffusion and reactions at surfaces and interfaces; theory of alloys; "order-N" algorithms; materials physics software.

- Mössbauer Studies-general studies, recent developments, novel uses, industrial applications.

- Fullerenes and Fullerene-Related Materials-experimental and theoretical research into physical properties of fullerenes, fullerene-derived compounds and composites, and carbon nanotubes.

- Avalanches, Earthquakes, and Fracture -experimental and theoretical approaches to the relationships among these traditionally separate subjects.

- Interaction of Crystal Lattice Defects and Superconductivity - to bring together researchers interested in the effect of defects in controlling and improving superconducting properties with researchers interested in the physics and materials science of defects.

- Diamond Growth, Properties, and Applications-materials (including related wide bandgap materials) range from high-purity single crystals to CVD films, covering pure and applied research.

- Magnetic Heterostructures, Thin Films, and Surfaces-emphasis on magnetotransport in multilayers and granular systems, quantum-well states in metallic layers and superlattices, interlayer coupling in transition-metal and rare-earth structures, and surface and interfacial anisotropy.

- Materials Research for Integrated Photonics-to bring together device researchers in guided-wave optics and physicists studying optical materials; new applications for nontraditional materials; fundamental science issues and opportunities to be resolved for the next generation of devices.

- Light Emission from Nanoscale Silicon Structures-fundamental electronic, optical, structural, and device properties of nanoscale silicon structures, including porous silicon and structures produced by other techniques.

- Phase Transformations-experimental, theoretical, and simulation studies in many different materials.

- Conducting Polymers-theory, experiment, and application.

- Epitaxy and Surface Morphologyphysics of epitaxial growth; surface morphology.

- Interfaces-experimental, theoretical, and simulation studies of solid-solid interfaces; effects of interfaces on physical properties of materials.

- Materials at High Pressure-recent developments in experimental and theoretical studies, including static and dynamic techniques.

- Laser Ablation and Thin-Film Deposition-fundamental aspects of laser ablation and its use in thin-film deposition; formation of novel materials.

- Semiconductor Surface Processinggroup IV and compound semiconductors; emphasis on treatments with technological implications such as substrate cleaning and preparation for epitaxial growth.

- Ferroelectric and Piezoelectric Materials-principles and applications of ferroelectric thin layers; experimental and phenomenological studies of ferroelectric materials; fundamental and theoretical studies.

- Clusters and Cluster-Assembled Materials-emphasis on physical, chemical and biological synthesis, characterization, and electronic, magnetic, optical, and mechanical properties.

- Structure and Properties of Noncrystalline Materials-recent advances achieved by a combination of experimental and theoretical techniques probing atomic and electronic structure, dynamics, and physical properties.

For more information about the APS meeting, or to register, contact: American Physical Society, One Physics Ellipse, College Park, MD 20740-2999; phone (301) 209-3285

\section{Preview: \\ 1994 MRS \\ Spring Meeting and Exhibit.} See p. 49-59 in this issue. 